\title{
Knee Osteochondritis Dissecans Treated by the AO Hook Fixation System: A Four Year Follow-Up of an Alternative Technique
}

\author{
Ioannis P. Pengas ${ }^{*}, 1,3$, Angelos Assiotis ${ }^{2}$, Michail Kokkinakis ${ }^{4}$, Wasim S. Khan ${ }^{1}$, Paul Meyers ${ }^{3}$, \\ James Arbuthnot ${ }^{5}$ and Michael J. Mcnicholas ${ }^{6}$
}

\author{
${ }^{1}$ Joint Reconstruction Unit, Royal National Orthopaedic Hospital, Stanmore, Middlesex, HA7 4LP, UK \\ ${ }^{2}$ Department of Orthopaedics and Trauma Surgery, Central Middlesex Hospital, Acton Lane, London, NW10 7NS, UK \\ ${ }^{3}$ Department of Orthopaedics and Trauma Surgery, Warrington Hospital, Lovely Lane, Cheshire, WA5 1QG, UK \\ ${ }^{4}$ Department of Orthopaedics and Trauma Surgery, Evelina Childrens Hospital, Guy's \& St Thomas Hospital NHS \\ Trust, Westminster Bridge Road, London, SE1 7EH, UK \\ ${ }^{5}$ Department of Orthopaedics and Trauma Surgery, Heart of England NHS Foundation Trust, Bordesley Green East, \\ Birmingham, B9 5SS, UK \\ ${ }^{6}$ Department of Orthopaedics and Trauma Surgery, Aintree University Hospital, Liverpool, Merseyside L9 7AL, UK
}

\begin{abstract}
Surgical fixation is recommended for stable osteochondritis dissecans (OCD) lesions that have failed nonoperative management and for all unstable lesions. In this study we set out to describe and evaluate an alternative method of surgical fixation for such lesions. Five knees with unstable OCD lesions in four male adolescent patients with open physes were treated with the AO Hook Fixation System. The outcome was evaluated both clinically and with three separate outcome systems (IKDC 2000, KOOS, Lysholm) at one and a mean four year follow-up. We demonstrated excellent clinical results in all patients. At four years, all scoring systems demonstrated statistically significant improvement when compared to the preoperative status. Our study suggests that the AO Hook Fixation System is an alternative method of surgical intervention with comparable medium term results with other existing modes of fixation and the added biomechanical advantage of the absence of distracting forces during hardware removal.
\end{abstract}

Keywords: AO hook fixation system, knee, osteochondritis dissecans.

\section{INTRODUCTION}

Osteochondritis Dissecans (OCD) is a term used to describe the separation of an articular cartilage subchondral bone segment from the remaining articular surface. OCD most commonly occurs in the knee, with the medial femoral condyle (MFC) being the most frequently affected site. The aetiology remains a matter of controversy and the same applies for treatment. Causative mechanisms have been grouped into constitutional (genetic), vascular and traumatic [1]. Injury to the subchondral plate results in de-stabilization of the overlying articular cartilage and increasing susceptibility in stress and shear forces. This eventually leads to fragmentation, loss of articular integrity and early degenerative changes.

The prevalence of OCD is estimated at 15-30 cases per 100,000 [1]. Males are affected 3 times more often than females and $30-40 \%$ of cases are bilateral with the average age at presentation being 10-20 years old. Plain radiography, $\mathrm{CT}, \mathrm{MRI}$ as well as direct visual inspection with arthroscopy have been used for the diagnosis, evaluation and classification

*Address correspondence to this author at the Joint Reconstruction Unit, Royal National Orthopaedic Hospital, Stanmore, Middlesex, HA7 4LP, UK; Tel: 07545083161; E-mail: yiannispengas@yahoo.com of knee OCD. Most classifications show the progression of the disease in stages.

There is a general consensus that undisplaced, stable OCD lesions on both clinical and radiological examination in younger patients should initially be treated non-operatively, as they have greater healing potential with reduced likelihood of further displacement and damage to the articular surface [2]. It was previously recommended that a period of non-weight-bearing and knee immobilization was the optimal treatment, but recently this has been replaced by restriction of sporting activities without immobilization or change in weight bearing status [3]. Stable lesions of the inferior-central medial femoral condyle demonstrate a greater healing success rate when compared with the lateral femoral condyle or the intercondylar medial femoral condyle [4].

Operative intervention is recommended for stable OCD lesions that failed nonoperative management and for all unstable lesions [5]. The internal fixation of isolated, salvageable, unstable OCD lesions represents the ideal initial mode of surgical treatment. We present the clinical follow up, at an average of 4 years after internal fixation of such lesions by the AO Hook Fixation System (Synthes ${ }^{\circledR}$ ) on five knee adolescent joints with open physes and we suggest that this method is an effective alternative to other existing 
methods. This method was initially developed and reported by Professor Roli Jakob, for the treatment of adult osteochondral fractures [6].

\section{PATIENTS AND SURGICAL METHOD}

The AO OCD Hook Fixation System $\left(\right.$ Synthes $^{\circledR}$ ) requires a 2-stage procedure: the first involves the reduction and stabilization of any displaced fragment that is salvageable and the second is for implant removal.

An arthroscopy is performed via anteromedial and anterolateral portals, to define the lesion's amenability for AO OCD Hook fixation and confirm the diagnosis. The AO Hooks (usually 2-3, $1.6 \mathrm{~mm}$ Hook wires) are passed through an accessory anterior portal, or via an arthrotomy incision, under direct vision and utilization of an image intensifier. The wire is directed distal to the physis. Each wire's position is checked with the image intensifier prior to advancement in a retrograde fashion with an air-powered drill until the Hook end is abutting the lesion. As the wire passes through the femoral condyle its sharp end tents the skin requiring stab skin incisions to facilitate access to the threaded extraarticular part of the wire which is used to achieve fragment compression via an embedding screw and nut combination. This secures the Hook in place and compresses the OCD lesion into its bed. The wires are then cut flush and the skin incision of approximately $4 \mathrm{~cm}$ is closed.

Postoperatively, continuous passive motion is employed for 48 hours, through the maximum range of knee movement tolerated. The patients are mobilized non-weight bearing for the first six weeks and full weight bearing is allowed thereafter. Return to sporting activities is delayed until radiographic union of the lesion is apparent and the wires are removed. This is done in a retrograde manner via the previous extra-articular incision by causing Hook straightening and thus allowing implant removal along with simultaneous arthroscopic visualization of the lesion.

We performed surgical fixation of unstable OCD lesions on five adolescent knee joints (4 patients) with open physes, using the AO Hook fixation system. The operations were all performed by the same senior surgeon $(\mathrm{MMcN})$. All 4 patients were male, with their OCD lesions identified over the lateral aspect of their medial femoral condyle. They had open physes at the time of operation (mean age 15.6, range 13-18 years of age). One 15-year old patient had bilateral disease and both knees were treated simultaneously. All patients had acute onset of symptoms after a twisting/ sporting injury; all complained of mechanical instability (Table 1).

The diagnosis was made initially with plain radiographs of the knee joints followed by MRI scans (Fig. 1) and confirmed intraoperatively. Surgery was carried out to stabilize the OCD lesions at a mean of 5.6 weeks after diagnosis of OCD ( 2 to 9 weeks). In all cases the AO Hook fixation system was used. The average lesion size was $2.0 \times 2.3 \mathrm{~cm}(2 \times 2$ to $2 \times 3 \mathrm{~cm})$. Two AO Hooks were used to fix four OCD unstable lesions while in one case three AO Hooks were used.

Follow-up appointments were arranged at 2, 6 and 12 weeks after operation to assess wound healing, range of motion and radiographic appearance (Fig. 2). The removal of the metalwork was performed at an average of 24 weeks ( 8 to 40 weeks) after confirmation of radiographic union. Subjective evaluation was carried out with the KOOS, IKDC and Lysholm scoring systems [7-10] preoperatively and at 4 years post fixation for all knees. Data were analyzed and statistical significance determined with the SPSS 17.0 software.

\section{RESULTS}

Second-look arthroscopies when removing the AO Hooks demonstrated integrity of the articular surface in all cases. We observed one case with a superficial extraarticular infection at the femoral wound site which was successfully treated with oral antibiotics and temporary haemarthrosis which settled down following knee aspiration. There were no cases of septic arthritis.

All AO OCD Hooks were removed once radiological union was evident. The two cases described above resulted in delayed removal of the Hooks at 36 and 40 weeks resulting on average time to removal of metalwork of 24 weeks after insertion. All patients returned to their pre-injury status with symmetrical range of movement and returned to sport at 12 months.

At 4 years all 3 scoring systems utilised demonstrated improvement when compared to the preoperative status. The average Lysholm knee score improved from 50.2 to 98 , the KOOS score from 58.9 to 97.1 and the IKDC score from 45.8 to 96.1 . All three scores increased to a statistically significant degree, as demonstrated with the use of the

Table 1. Demographic data and symptoms.

\begin{tabular}{|c|c|c|c|c|}
\hline Patient ID & Age & Site & Mode of Injury & Presentation \\
\hline \hline A & 13 & L & Rugby & Giving way and locking \\
\hline B & 15 & R & Football & Giving way \\
\hline C & 15 & L & Football & Giving way \\
\hline D & 17 & L & Football & Giving way \\
\hline E & 18 & L & Twisting / ADL & Giving way \\
\hline F & 31 & R & Squash & Swelling, giving way and $\downarrow$ ROM \\
\hline G & 32 &
\end{tabular}

$\mathrm{ADL}=$ Activities of Daily Living. 


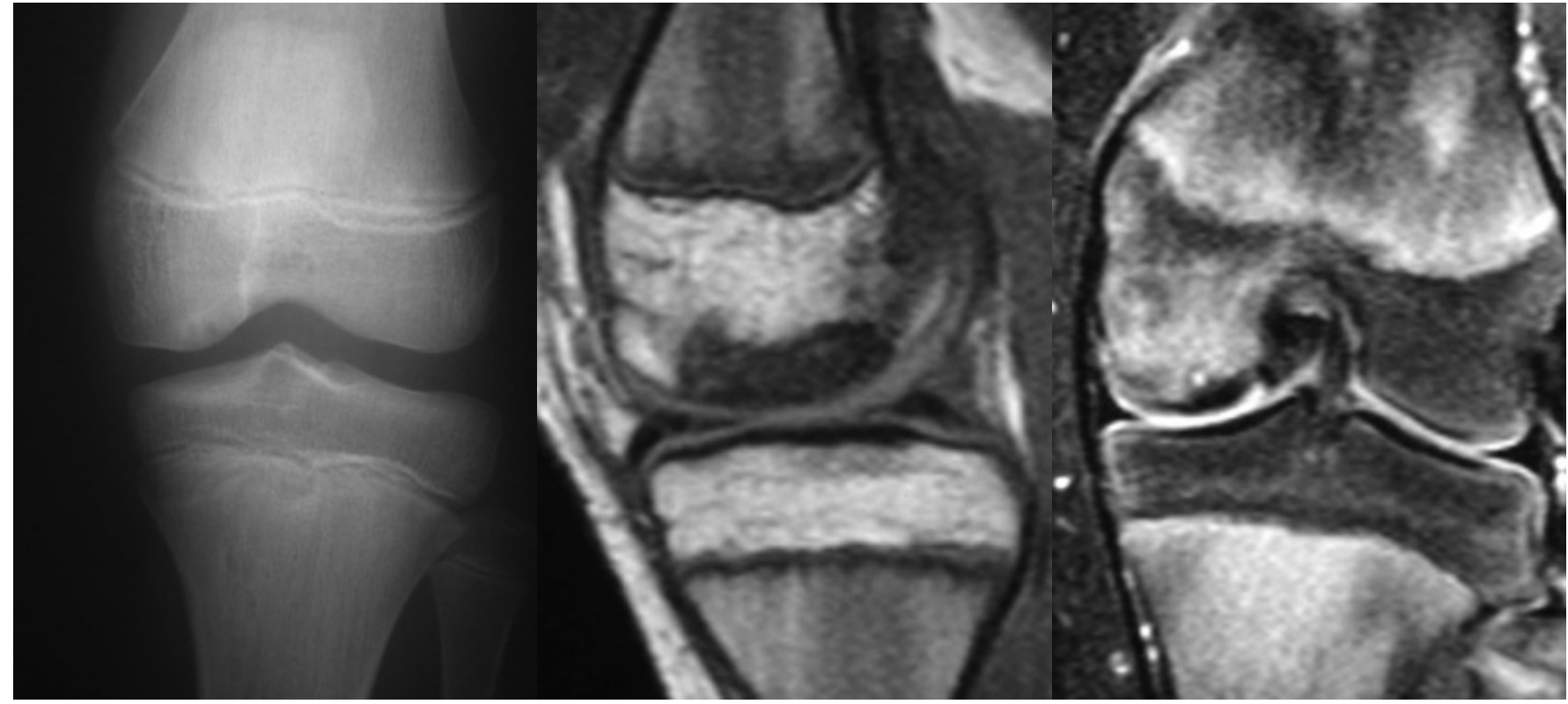

Fig. (1). Preoperative radiographic imaging of the same knee: Anteroposterior radiograph (a), sagittal T2 MRI image (b), coronal T2 MRI image (c).

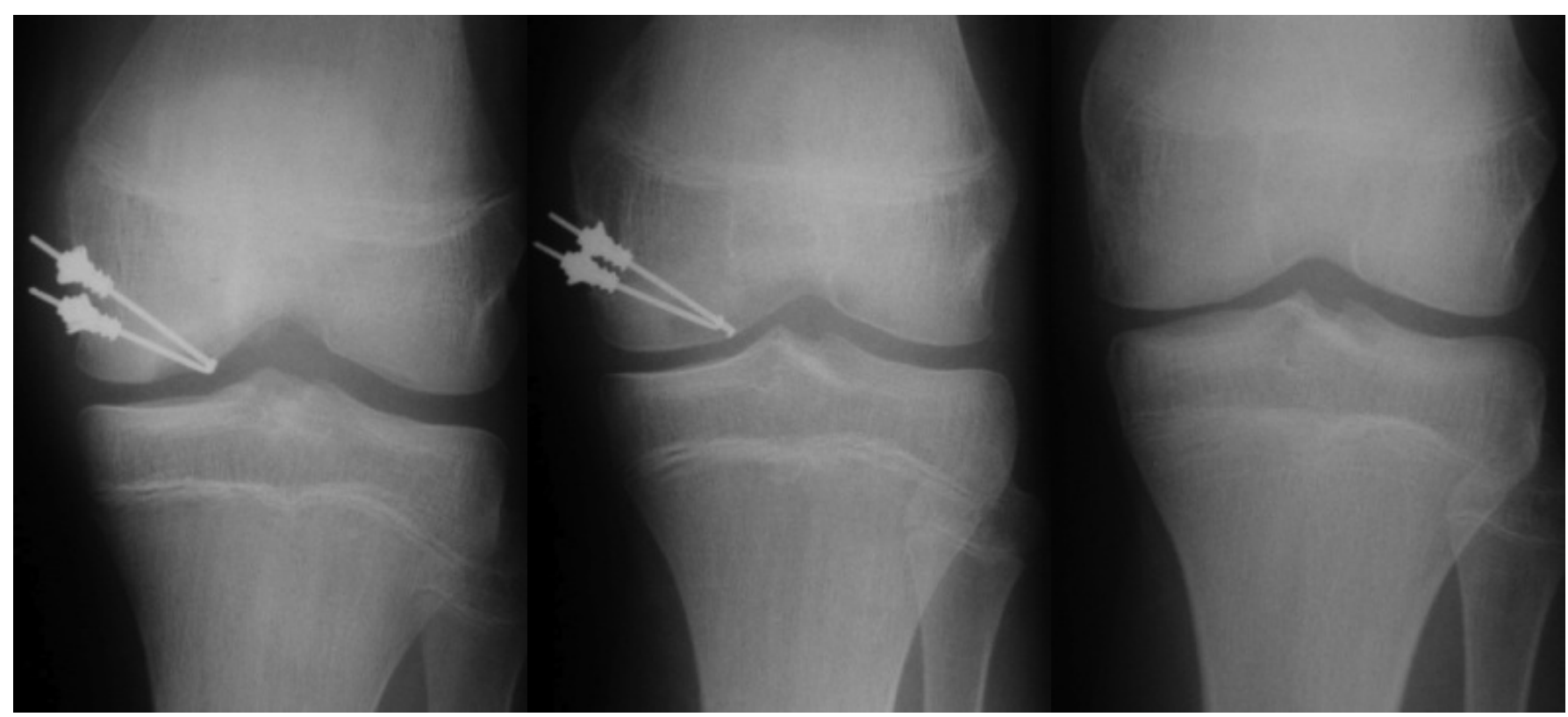

Fig. (2). Postoperative radiographic imaging of the same knee: Anteroposterior radiograph 2 weeks postoperatively (a), 12 weeks postoperatively (b), and after metalwork removal at 28 weeks (c).

Wilcoxon signed rank test. The p-values (with $95 \%$ confidence intervals) were $<0.05$ for all three scoring systems (Table 2). All four patients demonstrated excellent clinical results. It is worth noting that the two patients who were not back to sport at the four year mark have declared lack of confidence and back pain in their questionnaires and not knee pain as a causative factor (Table $\mathbf{3}$ ).

\section{DISCUSSION}

Current treatment modalities in juvenile OCD are present with variable results in current literature. We present an alternative method that results in stable fixation and at the same time, is less destructive upon metalwork removal. Juvenile OCD is differentiated from the adult form by observing whether the physis of the involved bone is open or 
Table 2. Knee scores for the 4 patients, preoperatively and postoperatively (p value $<0.05$ ).

\begin{tabular}{|c|c|c|c|c|c|c|}
\hline \multirow{2}{*}{ Patient ID } & \multicolumn{3}{|c|}{ Preoperative Score } & \multicolumn{3}{|c|}{ Postoperative Score at 4 Years } \\
\cline { 2 - 7 } & KOOS & IKDC & Lysholm & KOOS & \multicolumn{2}{|c|}{ IKDC } \\
\hline \hline A & 73.214 & 52.9 & 46 & 100 & 100 & 100 \\
\hline B & 67.900 & 52.9 & 68 & 96.428 & 96.5 & 96.5 \\
\hline C & 70.238 & 57.5 & 74 & 96.428 & 98.8 & 100 \\
\hline D & 46.428 & 39.1 & 33 & 97.023 & 95 & 98.5 \\
\hline E & 36.904 & 26.4 & 30 & 95.833 & 95 \\
\hline
\end{tabular}

Table 3. Postoperative symptoms and return to sports.

\begin{tabular}{|c|c|c|c|c|c|c|}
\hline B & Monthly & Not at all & Not at all & None & Yes & Yes \\
\hline $\mathrm{C}$ & Monthly & Not at all & Not at all & None & Yes & Yes \\
\hline E & Never & Mildly & Not at all & None & Yes & No (back pain) \\
\hline
\end{tabular}

closed at the time of presentation. Both forms of OCD are different entities with different management, strategies and prognoses.

There is a plethora of different techniques described for the management of OCD lesions. The modality of treatment depends on a variety of factors such as the chronic or acute character of the injury (period between symptoms-onset and treatment), the age and activity level of the patient, the configuration of the lesion (size, location, stability), the presence of extensive cartilage disease and other factors including malalignment, meniscus tear, anterior cruciate ligament rupture and patella mal-tracking [11-13].

Unstable OCD lesions are characterized by partial or full detachment of the fragment from its subchondral bed and fall into two categories: salvageable or unsalvageable (congruous $v s$ incongruous articular surface), this in effect directing fixation or removal [14].

Internal fixation of unstable, salvageable and isolated OCD lesions provides stability of the osteochondral fragment in its subchondral bed and offers the advantage of preserving a congruous articular surface [15]. Excision of unstable OCD lesions is associated with poor long-term results as the damaged articular surface can lead to early osteoarthritis $[16,17]$. Promising future modes of treatment have emerged such as tissue engineering products, smart scaffolds, bioreactors, genetics and growth factors $[18,19]$.

When internal fixation is combined with simultaneous bone marrow stimulation by ante- or retrograde drilling of the subchondral bed, aggressive healing response is often promoted, particularly in younger patients [20]. Autologous cancellous graft can be used in large-sized OCD lesions with possible bony defects, in order to prevent subsidence of the osteochondral fragment. The unstable osteochondral fragment can be fixed using bone pegs [21], metallic or absorbable pins or screws [22], metal staples [23], combination of pins and screws or using the AO Hook system.

Moderate to good clinical results have been shown when securing OCD lesions with autologous cortical bone peg fixation [21] and arthroscopic mosaicplasty [24]. Kivisto et al. used metallic staples in 28 knees with OCD lesions and reported metalwork breakage in 9 knees and complete healing in only 14 cases [23].

Wouters et al. suggested the use of drilling and fixation of OCD lesions with bioabsorbable pins for stable lesions and the combination of a metallic screw with absorbable pins for unstable OCD defects [25]. Din et al. reported excellent results in 12 knees [26] and Dines et al. in 7 out of 9 knees at a mean follow up of 2.6 years [22], both using bioabsorbable pin fixation of OCD lesions. Nakagawa et al. also reported good or excellent results in 7 out of 8 knees using bioabsorbable pins [27]. Despite reports of good clinical results using bioabsorbable screws there have been reports of inflammatory reactions, backing out and breakage of such implants [28].

Cugat et al. reported a percentage of $93 \%$ of good or excellent results in 15 knees treated with arthroscopically placed cannulated screws [29]. Kouzelis et al. reported good results 9 of 10 patients at 2.2 years follow up using Herbert screw fixation combined with retrograde drilling of the subchondral bed [30]. Makino et al. performed radiological (MRI), arthroscopical and clinical evaluation of 15 knees at a mean follow up of 4.1 years, reporting that all but one knees had evidence of stable fixation with an intact smooth surface after using arthroscopic Herbert screw fixation [31]. 
Magnussen et al. reported normal function in activities of daily living in 12 patients treated with metallic screw fixation for OCD loose bodies at 9 years follow up [32]. Herbert screw fixation seems to have good results, but potential metalwork removal may require an osteochondral plug to be "cored out", damaging an area of articular cartilage [33]. A biomechanical study by Morelli et al. has shown that pin fixation of OCD lesions offers resistance against shear loading and stability improved with the use of long implants that compressed the fragments together [34]. An example of such a device is the AO Hook system [6].

A second operation to remove the metalwork is certainly one of the disadvantages of non-biodegradable fixation systems, such as the AO Hook system, but a good fixation and compression of the unstable OCD lesions can be achieved. Arguably the main advantage of this technique is the lack of cartilage "core-out", during implant removal.

We have demonstrated excellent clinical results in 5 knees, 4 years after fixation of unstable OCD lesions in adolescent patients using the AO Hook fixation system. As far as we are aware this technique has not been previously described in the English literature. Amongst the many operative techniques that achieve stable fixation of OCD lesions, the AO Hook fixation system seems to be an alternative procedure leading to good medium term results.

However, we acknowledge that the small number of cases is a limitation of this study but would like to point out that it is the biggest series with this fixation device, demonstrating results consistent with the international literature. Furthermore, we have subsequently learned from the manufacturer that commercialization of this implant was stopped for unrevealed reasons.

\section{CONFLICT OF INTEREST}

The authors confirm that this article content has no conflict of interest.

\section{ACKNOWLEDGEMENTS}

Declared none.

\section{REFERENCES}

[1] Crawford DC, Safran MR. Osteochondritis dissecans of the knee. J Am Acad Orthop Surg 2006; 14(2): 90-100.

[2] Hughston JC, Hergenroeder PT, Courtenay BG. Osteochondritis dissecans of the femoral condyles. J Bone Joint Surg Am 1984; 66(9): 1340-8

[3] Lefort G, Moyen B, Beaufils P, et al. [Osteochondritis dissecans of the femoral condyles: report of 892 cases]. Rev Chir Orthop Reparatrice Appar Mot 2006; 92(5 Suppl): 2S97-141.

[4] Yoshida S, Ikata T, Takai H, Kashiwaguchi S, Katoh S, Takeda Y. Osteochondritis dissecans of the femoral condyle in the growth stage. Clin Orthop Relat Res 1998; (346): 162-70.

[5] Polousky JD. Juvenile osteochondritis dissecans. Sports Med Arthrosc 2011; 19(1): 56-63.

[6] Jakob RP, Miniaci A. A compression pinning system for osteochondritis dissecans of the knee. Acta Orthop Scand 1989; 60(3): 319-21.
[7] Roos EM, Toksvig-Larsen S. Knee injury and Osteoarthritis Outcome Score (KOOS) - validation and comparison to the WOMAC in total knee replacement. Health Qual Life Outcomes 2003; 1: 17.

[8] Roos EM, Roos HP, Lohmander LS, Ekdahl C, Beynnon BD. Knee Injury and Osteoarthritis Outcome Score (KOOS)--development of a self-administered outcome measure. J Orthop Sports Phys Ther 1998; 28(2): 88-96.

[9] Hefti F, Muller W, Jakob RP, Staubli HU. Evaluation of knee ligament injuries with the IKDC form. Knee Surg Sports Traumatol Arthrosc 1993; 1(3-4): 226-34.

[10] Tegner Y, Lysholm J. Rating systems in the evaluation of knee ligament injuries. Clin Orthop Relat Res 1985; (198): 43-9.

[11] Amin AA BW, Gooding CR et al. The use of autologous chondrocyte implantation following and combined with anterior cruciate ligament reconstruction. Int Orthop 2006; 30: 48-53.

[12] Gomoll AH, Minas T, Farr J, Cole BJ. Treatment of chondral defects in the patellofemoral joint. J Knee Surg 2006; 19(4): 28595 .

[13] Szerb I, Hangody L, Duska Z, Kaposi NP. Mosaicplasty: long-term follow-up. Bull Hosp Jt Dis 2005; 63(1-2): 54-62.

[14] Detterline AJ, Goldstein JL, Rue JP, Bach BR, Jr. Evaluation and treatment of osteochondritis dissecans lesions of the knee. J Knee Surg 2008; 21(2): 106-15.

[15] Kocher MS, Czarnecki JJ, Andersen JS, Micheli LJ. Internal fixation of juvenile osteochondritis dissecans lesions of the knee. Am J Sports Med 2007; 35(5): 712-8.

[16] Anderson AF, Pagnani MJ. Osteochondritis dissecans of the femoral condyles. Long-term results of excision of the fragment. Am J Sports Med 1997; 25(6): 830-4.

[17] Wright RW, McLean M, Matava MJ, Shively RA. Osteochondritis dissecans of the knee: long-term results of excision of the fragment. Clin Orthop Relat Res 2004; (424): 239-43.

[18] Stoop R. Smart biomaterials for tissue engineering of cartilage. Injury 2008; 39 (Suppl 1): S77-87.

[19] Gaissmaier C, Koh JL, Weise K, Mollenhauer JA. Future perspectives of articular cartilage repair. Injury 2008; 39 (Suppl 1): S114-20.

[20] Kocher MS, Tucker R, Ganley TJ, Flynn JM. Management of osteochondritis dissecans of the knee: current concepts review. Am J Sports Med 2006; 34(7): 1181-91.

[21] Slough JA, Noto AM, Schmidt TL. Tibial cortical bone peg fixation in osteochondritis dissecans of the knee. Clin Orthop Relat Res 1991; (267): 122-7.

[22] Dines JS, Fealy S, Potter HG, Warren RF. Outcomes of osteochondral lesions of the knee repaired with a bioabsorbable device. Arthroscopy 2008; 24(1): 62-8.

[23] Kivisto R, Pasanen L, Leppilahti J, Jalovaara P. Arthroscopic repair of osteochondritis dissecans of the femoral condyles with metal staple fixation: a report of 28 cases. Knee Surg Sports Traumatol Arthrosc 2002; 10(5): 305-9.

[24] Miniaci A, Tytherleigh-Strong G. Fixation of unstable osteochondritis dissecans lesions of the knee using arthroscopic autogenous osteochondral grafting (mosaicplasty). Arthroscopy 2007; 23(8): 845-51.

[25] Wouters DB, Bos RR, van Horn JR, van Luyn MJ. Should in the treatment of osteochondritis dissecans biodegradable or metallic fixation devices be used? A comparative study in goat knees. J Biomed Mater Res B Appl Biomater 2008; 84(1): 154-64.

[26] Din R, Annear P, Scaddan J. Internal fixation of undisplaced lesions of osteochondritis dissecans in the knee. J Bone Joint Surg Br 2006; 88(7): 900-4

[27] Nakagawa T, Kurosawa H, Ikeda H, Nozawa M, Kawakami A. Internal fixation for osteochondritis dissecans of the knee. Knee Surg Sports Traumatol Arthrosc 2005; 13(4): 317-22.

[28] Scioscia TN, Giffin JR, Allen CR, Harner CD. Potential complication of bioabsorbable screw fixation for osteochondritis dissecans of the knee. Arthroscopy 2001; 17(2): E7.

[29] Cugat R, Garcia M, Cusco X, et al. Osteochondritis dissecans: a historical review and its treatment with cannulated screws. Arthroscopy 1993; 9(6): 675-84. 
[30] Kouzelis A, Plessas S, Papadopoulos AX, Gliatis I, Lambiris E. Herbert screw fixation and reverse guided drillings, for treatment of types III and IV osteochondritis dissecans. Knee Surg Sports Traumatol Arthrosc 2006; 14(1): 70-5.

[31] Makino A, Muscolo DL, Puigdevall M, Costa-Paz M, Ayerza M. Arthroscopic fixation of osteochondritis dissecans of the knee: clinical, magnetic resonance imaging, and arthroscopic follow-up. Am J Sports Med 2005; 33(10): 1499-504.

[32] Magnussen RA, Carey JL, Spindler KP. Does operative fixation of an osteochondritis dissecans loose body result in healing and long- term maintenance of knee function? Am J Sports Med 2009; 37(4): 754-9.

[33] Rey Zuniga JJ, Sagastibelza J, Lopez Blasco JJ, Martinez Grande M. Arthroscopic use of the Herbert screw in osteochondritis dissecans of the knee. Arthroscopy 1993; 9(6): 668-70.

[34] Morelli M, Poitras P, Grimes V, Backman D, Dervin G. Comparison of the stability of various internal fixators used in the treatment of osteochondritis dissecans--a mechanical model. J Orthop Res 2007; 25(4): 495-500.

(C) Pengas et al.; Licensee Bentham Open.

This is an open access article licensed under the terms of the Creative Commons Attribution Non-Commercial License (http://creativecommons.org/licenses/by-nc/3.0/) which permits unrestricted, non-commercial use, distribution and reproduction in any medium, provided the work is properly cited 